\title{
A Modified Real-Time Fault-Tolerant Task Allocation Scheme for Wireless Sensor Networks
}

\author{
F. F. Marshall ${ }^{* 1}$, M. B. Mu'azu' , I. J. Umoh ${ }^{3}$, A. T. Salawudeen ${ }^{4}$, B. O. Sadiq ${ }^{5}$, D. E. Ikpe ${ }^{6}$ \\ 1,2,3,4,5,6 Ahmadu Bello University/Department of Computer Engineering \\ frankdidam14@yahoo.com ${ }^{*}$
}

\begin{abstract}
In WSNs, the sensor nodes are at risk of failure and malicious attacks (selective forwarding). This may have a profound negative effect when you consider real-time WSNs, making them challenging to deploy. When there is a delay in tasks allocation execution processes in real-time WSNs because of sensor nodes failures, this will cause disastrous consequences if the systems are safety-critical, e.g. aircraft, nuclear power plant, forest fire detection, battlefield monitoring, thus the need to developed a real-time system that is fault-tolerable. This paper developed a modified real-time fault-tolerant task allocation scheme (mRFTAS) for WSNs (wireless sensor networks), using active replication techniques. mRFTAS and RFTAS performance were compared using time of execution of the task, network lifetime and reliability cost. The mRFTAS performance showed an improvement over that of RFTAS when it comes to reducing the time it takes for task execution by $45.56 \%$ and reliability cost of $7.99 \%$ while prolonging the network lifetime by $36.35 \%$.
\end{abstract}

Keywords: WSNs, Real-time, Fault-tolerant, Task Allocation

\section{Introduction}

A WSN comprises of numerous resource-constrained sensor nodes that, are frequently deploy in unattended environments. Consequently, the sensor nodes are at risk of failure and/or malicious attacks. The failed nodes can have a profoundly negative effect on real-time WSNs. WSNs have a major constraint, which is the low power consumption requirement of sensor nodes[1][2]. Parallel processing between sensor nodes is an innovative technology, which supports the essential computation capacity in WSNs while ensuring low power consumption by the sensor nodes.

A key issue to consider in real-time systems is the time delay in task processing (which can arise from issues associated with nodes failures). It is crucial that network failures are identify early, and the proper actions taken, to ensure network reliability and network lifetime is prolong.

Task allocation plays a significant role in parallel processing. Assigning a task to the suitable sensor nodes and simultaneously balancing the network load in the context of the uncertain and dynamic network environments are essential to parallel processing[1]. In WSNs, the issue of task allocation involves assigning tasks logically within sensor nodes, to reduce the general power utilization while the tasks are complete before the set deadlines, thus prolonging the lifetime of the sensor network [2][3][4]. Load balancing is an essential factor for prolonging the network lifetime. In the absence of proper task allocation techniques, every sensor node will work independently of others [5]. WSNs have challenges such as instability of wireless communication links and dynamically changing topologies, there are potentially additional uncertainties and vulnerabilities for real-time applications. A sensor node failure should not necessarily affect the entire tasks processing of the sensor network, especially for safety and security critical applications [6].

In order to sustain the performance of the sensor networks without interruption due to failures of the sensor node, fault tolerance turns out to be a valuable initiative. For instance, if sensor nodes are being deploy in a battlefield or military-camp for surveillance and detection, the fault tolerance has to be high because the sensed data are critical for security and safety reasons. Hence, a fault-tolerant mechanism is mandatory for such safety and time-critical applications [7] [8].

Marshall, F., Mu'azu, M., Umoh, I., Salawudeen, T., Sadiq, B., \& Ikpe, D. (2018). A Modified RealTime Fault-Tolerant Task Allocation Scheme for Wireless Sensor Networks. Kinetik: Game Technology, Information System, Computer Network, Computing, Electronics, and Control, 4(1). doi:http://dx.doi.org/10.22219/kinetik.v4i1.659

Receive June 11, 2018; Revise August 03, 2018; Accepted August 30, 2018 
The replication backup techniques which involves the use of primary/backup (P/B) system is the widely used technique for fault-tolerant task allocation as it tolerates copies of a task to be processed on separate sensor nodes.

There is a need for a real-time WSN that is fault-tolerant and safety-critical, thus the need for the development of a modified real-time fault-tolerant task allocation scheme (mRFTAS), such as to avoid the breakdown of a system due to failure of some sensor nodes.

Literature reviewed show that research has been carried in the area of task allocation/scheduling schemes, and fault tolerant task allocation scheme in WSNs. Successful task allocation execution in systems that are real-time with little or no delay in task processing time, reduced task waiting time is still an open-ended research area for real-time WSNs.

The real-time fault-tolerant task allocation scheme (RFTAS) is the kind of scheme that is used to prevent system failure or system breakdown and has mostly employed the passive replication backup technology [9]. However, the passive replication scheme has the problem of delay in task processing time. Delay in task processing time can be disastrous for real-time systems that are critical in term of safety.

This research developed a modified real-time fault-tolerant task allocation scheme (mRFTAS) using the active replication backup scheme, to address the issue of delay in task processing time associated with the RFTAS.

The modified real-time fault-tolerant task allocation scheme will help a system to keep operating even in the presence of some failures, inevitably reducing the total time of an entire task allocation process, thus prolonging the network lifetime.

\section{Research Method}

\subsection{Total Time of Task Execution}

The total execution time to finish all tasks depends on the time of execution the primary copies and the active backup copies, execution time for $m$ tasks and can be defined Equation 1 [2].

$$
T=\sum_{i=1}^{m} e t_{i, p\left(t_{i}^{P}\right)}+\sum_{i=1}^{m} S\left(t_{i}^{B}\right) \times\left(t_{i}^{B}-l s t_{i, p\left(t_{i}^{B}\right)}\right)
$$

Where, $T$ is time, $S\left(t_{i}^{B}\right)$ represents the backup mode $\left(t_{i}^{B}\right)$. If $l_{i}$ is not less than the $\left(e t_{i, p\left(t_{i}^{B}\right)}\right)$, $t_{i}^{B}$ need not to be execute before $\left(f_{i}^{P}\right)$ for fault-tolerant. Even $n_{S\left(t_{i}^{P}\right)}$ fails before the time $f_{i}^{P}, t_{i}^{B}$ also can be finished before $d_{i}$. Thus, it uses passive mode. Otherwise, $t_{i}^{B}$ will be carry before $f_{i}^{P}$ for fault-tolerant. Thus, it employs active mode. Let $S\left(t_{i}^{B}\right)$ is equal 1 , which means $t_{i}^{B}$ uses active backup mode. Otherwise, $t_{i}^{B}$ uses passive mode. $S\left(t_{i}^{B}\right)$ will also mean as Equation 2 [2].

$$
S=\left\{\begin{array}{cc}
1, & \text { if }\left(l_{i}\left(t_{i}^{B}\right)<e t_{i, p\left(t_{i}^{B}\right)}\right) \\
0 & \text { else }
\end{array}\right.
$$

Generally, primary copy when executed successfully, the corresponding backup copy needs not to execute anymore.

\subsection{Reliability}

Reliability in WSNs is the probability that none of real-time tasks will failed due to sensor nodes failures in the network. Therefore, the reliability model is given similar to Equation 3,4,5 [2].

$$
\begin{gathered}
R C=100 \times(R C(z p)+R C(z b)) \\
R C(z p)=\sum_{i=1}^{m} \lambda_{p\left(t_{i}^{P}\right)} \times e t_{i, p\left(t_{i}^{P}\right)} \\
R C(z p)=\sum_{i=1}^{m} S\left(t_{i}^{B}\right) \times \lambda_{p\left(t_{i}^{P}\right)} \times\left(f_{i}^{P}-l s t_{i p\left(t_{i}^{B}\right)}\right)
\end{gathered}
$$




$$
R e l=\exp (-R C)
$$

where $R e$ l denotes the reliability in a network, $\mathrm{RC}$ denotes reliability cost, $R C(z p)$ and $R C(z b)$ are the total reliability costs of primary copy and a copy used for backup of $m$ tasks, and $\Lambda_{i}$ denotes the failure probability of $n_{i}$. Take Figure 1 as an example, if the failure probability of $n_{2}$ is relatively large and it also needs to work for some time; this clearly shows that system reliability will surely decline. Thus, network reliability decreases with the increase of the reliability cost as represented in Equation 6.

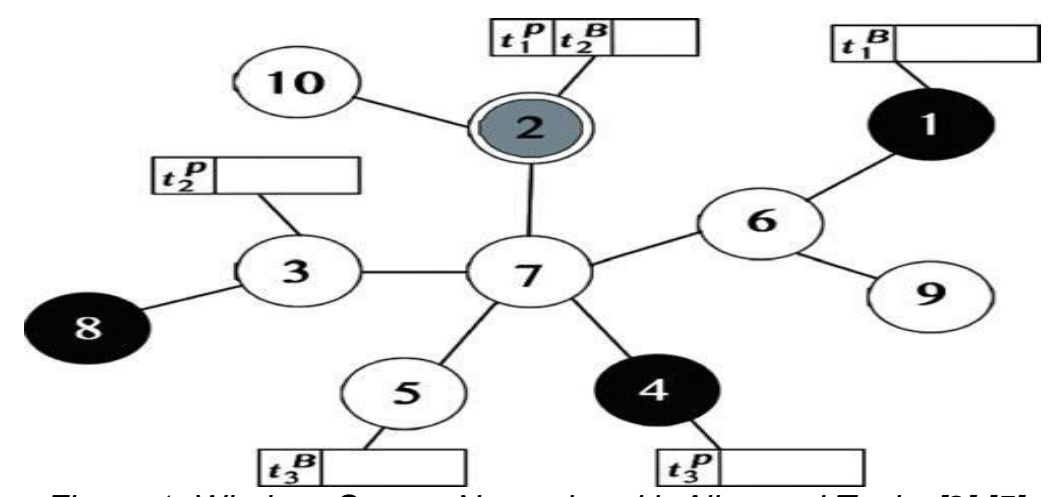

Figure 1. Wireless Sensor Networks with Allocated Tasks [2] [5]

Generally, let $m$ tasks be allocated satisfactorily to $n$ sensors in order to minimize task execution time, balance the network load, prolong the network lifetime, certify that the task would not fail by unexpected failure of nodes and improve the dependability of task supervision [3].

\subsection{Network Lifetime}

Task allocation is an optimization problem that can be formalized as seen Equation 7 [2].

$$
\operatorname{Maximize}(i t) S . T .=\left\{\begin{array}{l}
\sum_{i=1}^{n} y_{i} \leq n u m \times 20 \% \\
\forall t_{i} T, n_{p\left(t_{i}^{P}\right)} \neq n_{p\left(t_{i}^{P}\right)}
\end{array}\right.
$$

where It denotes WSNs lifetime and num symbolizes the number of sensor nodes total used. $y_{i}=1$ shows that node $n_{i}$ is a failure and $y_{i}=0$ indicate that node $n_{i}$ is normal. Assume that WSNs are paralyzed when a number of failure nodes exceed $20 \%$ of the total number. For example, if $n_{1}, n_{4}$, and $n_{8}$ break down in Figure 1 , then $\sum_{i=1}^{10} y_{i}=3$ is larger than $n u m \times 20 \%=2$, the network becomes paralyzed.

\subsection{Calculation Process of the Earliest Start Time of the Task's Primary Copy}

Let's assume there is a task $t_{j}$, the primary start time of $t_{j}^{P}$ which is designated to node $n_{i}$ can be calculated, we examine every node's idle time space $\left[0, S_{1}^{\prime}\right],\left[f_{1}^{\prime}, S_{2}^{\prime}\right],\left[f_{2}^{\prime}, S_{3}^{\prime}\right], \ldots,\left[f_{n}^{\prime},+\infty\right]$ from left to right, where $S_{i}^{\prime}$ and $f_{i}^{\prime}$ stands for the start time and finish time of the ith task in the task queue of $n_{i}$, respectively. For predictable expression, let $f_{0}^{\prime}=0$, In the event that the first idle time space $\left[f_{k}^{\prime}, S_{k+1}^{\prime}\right]$, which can meet $\max \left(a_{j}, f_{k}^{\prime}\right)+e t_{j . i} \leq d_{j}$, is discovered, the earliest start time of $t_{j}^{P}$ on node $n_{i}$ would be noted as $e s t_{j i}=f_{k}^{\prime}$ generally $e s t_{j i}$ would be noted as $+\infty$.

\subsubsection{Calculation Process of the Latest Start Time of the Task's Backup Copy}

Let us assume there is a task $t_{j}$, the primary start time of $t_{j}^{B}$ which is apportioned to node $n_{i}$ can be determined as follows: 
1. Step 1

Initialize time space $=\left[d_{j}-e t_{j i}, d_{j}\right]$, for comfort, the start time and finish time of space can be set apart as $s_{-}$start and $s_{-}$finish, i.e. space $=\left[s_{-}\right.$begin, $\left.s_{-} f i n d\right]$.

2. Step 2

Scan the task queue of $n_{i}$. If the slot is set in its idle times, let $l s t_{j i}$ be $s_{-} s t a r t$ and after that, the procedure is finished. Else, it implies that space overlaps with another task in the line, hence, stamp the overlapped task as $t_{x}$ for further consideration. In any case, if $t_{x}$ is a primary copy or an active backup copy, update space with $s_{-}$finish $=$the start time of $t_{x}$, $s_{-} s t a r t=s_{-}$finish $-e t_{j i}$. And after that, if $s_{-}$finish $<0$, let $l s t_{j i}$ be $+\infty$ and the procedure is finished, else goes to Step 2 once more.

\subsubsection{Allocation Process of the Task's Primary Copy}

Plan a utility function $U^{P}(i, j)$ to quantify the comprehensive execution of every node processing $t_{j}^{P}$ and allot $t_{j}^{P}$ to a superior node in Equation 8 [2].

$$
U^{P}(i, j)=w t_{1} \times U B(i, j)+w t_{2} \times U E(i, j)+w t_{3} \times U R(i, j),
$$

where $w t_{1}, w t_{2}$ and $w t_{3}$ are weight coefficient, $U^{P}(i, j)$ is the utility function of primary copy, the slighter the estimation of $U^{P}(i, j)$ is, the better $n_{i}$ execute $t_{j}^{P}$ completely. $U B(i, j)$, $U E(i, j)$ and $U R(i, j)$ signify the load degree, energy utilization degree and failure proportion level of $n_{i}$ which executing $t_{j}^{P}$ contrasted and different nodes which participate in $t_{j}$ The present load $b_{i}$, energy utilization ene $e_{j, i}$ and failure proportion $\lambda_{i}$ of $n_{i}$ are mapped in the scope of 0 and 0.5 by utilizing a comparable sigmoid function as information standardization function to achieve $U B(i, j), U E(i, j)$ and $U R(i, j)$. The specific estimation equations are presented by the following Equation 9, 10, 11 [2].

$$
\begin{aligned}
& U B(i, j)=\left\{\begin{array}{l}
0, \quad \text { if } \quad\left(b_{\max }-b_{\min }\right)=0 \\
\frac{1}{e-\frac{x_{j i} \times b_{i}-b_{\min }}{b_{\max }-b_{\min }+1}}-0.5, \text { else }
\end{array}\right. \\
& U E(i, j)=\left\{\begin{array}{l}
0, \quad \text { if } \quad\left(\text { ene }_{\text {max }}-\text { ene }_{\text {min }}\right)=0 \\
\frac{1}{e-\frac{x_{j i} \times \text { ene }_{i}-\text { ene }_{\text {min }}}{\text { ene } e_{\text {max }}-\text { ene }_{\text {min }}+1}}-0.5, \text { else }
\end{array}\right. \\
& U R(i, j)=\left\{\begin{array}{l}
0, \quad \text { if } \quad\left(\lambda_{\max }-\lambda_{\min }\right)=0 \\
\frac{1}{e-\frac{x_{j i} \times \lambda_{i}-\lambda_{\min }}{\lambda_{\max }-\lambda_{\min }+1}}-0.5
\end{array}\right.
\end{aligned}
$$

where $b_{\max }$ and $b_{\min }$ indicate the high load and the lightest load of nodes which take an interest in $t_{j}$, separately, the smaller the $U B(i, j)$, the lighter the load of $n_{i}$ when executing $t_{j}^{P}$ . ene $e_{\max }$, and $e n e_{\min }$ signify the biggest and the slightest energy utilization of nodes which take 
an interest in $t_{j}$, separately. The smaller the $U E(i, j)$, the less the energy utilization of $n_{i}$ when executing $t_{j}^{P} . \lambda_{\max }$, and $\lambda_{\min }$ mean the biggest and the minimum failure proportion of nodes which take an interest in $t_{j}$, separately. The two steps can present the specific allocation procedure of a task's primary copy:

1. Step 1.

For every node $n_{i}$ which takes part in $t_{j}$, calculate the total of $e s t_{j i}$, and $e t_{j i}$, for foreseeing time required of $t_{j}^{P}$ to finished on nodes $n_{i}$. In the event that the deadline requirement of $t_{j}$ is met, $U^{P}(i, j)$ will be calculated by Equation 8 . Generally, $U^{P}(i, j)$, will be noted $+\infty$ until each one of those nodes has taken into consideration.

2. Stage 2.

Select a node with the less $U^{P}(i, j)$, and afterwards allot $t_{j}^{P}$ to the chose node and update it's comparing $U^{P}(i, j)$ and $U B(i, j)$ for next estimation convenience.

\subsubsection{Allocation Process of the Task's Backup Copy}

\section{Step 1}

There exists a backup copy. For every node $n_{i}$ which takes part in $t_{j}$, compute the total of $l s t_{j i}$ and $e t_{j i}$ to determinate the perform method of reinforcement copy and forecast the completion time of $t_{j}^{B}$ on node $n_{i}$. In the event that the deadline requirement of $t_{j}^{B}$ is met, $U^{B}(i, j)$ will be computed using Equation 12, else, $U^{B}(i, j)$ will be observed as $+\infty$ pending when all nodes are taken into account.

2. Step 2

Choose a node with the least value of $U^{B}(i, j)$, assign $t_{j}^{B}$ to the chosen node and update the relating $U^{B}(i, j)$ and $U B(i, j)$ [2].

$$
\begin{gathered}
U E^{\prime}(i, j)=\left\{\begin{array}{c}
0, \quad \text { if } \quad\left(e n \dot{e}_{\text {max }}-e n \dot{e}_{\text {min }}\right)=0 \\
\frac{1}{e-\frac{x_{j i} \times S\left(t_{j}^{B}\right) \times e n e_{j i} \times \operatorname{per}_{j i} \times e n \dot{e}_{j i}}{e n \dot{e}_{\text {max }}-e n e_{\text {min }}+1}}-0.5
\end{array}\right. \\
U^{B^{\prime}}(i, j)=w t_{1} \times U B^{\prime}(i, j)+w t_{2} \times U E^{\prime}(i, j)+w t_{3} \times U R^{\prime}(i, j),
\end{gathered}
$$

Presented by Equation 13, where $\mathrm{wt}_{1}$, wt 2 and wt3, are weight coefficients, $U^{B}(i, j)$ is the utility function of reinforcement copy. The slighter the quantity of $U^{B}(i, j)$ is, the better $n_{i}$ execute $t_{j}^{B}$ widely. $e n e_{\min }^{\prime}$, and $e n e_{\max }^{\prime}$ represent the minimum and the maximum energy utilization of the nodes which take part in $t_{j}$, correspondingly. en $e_{\min }^{\prime}$ and $e n e_{\max }^{\prime}$ can be estimated by the following Equation 14 and 15 [2].

$$
\begin{aligned}
& \text { ené }_{\text {min }}=\min _{i=1}\left(x_{j i} \times S\left(t_{j}^{B}\right) \times \text { ene }_{j i} \times \text { per }_{j i}\right) \\
& \text { ené }_{\text {min }}=\max _{i=1}\left(x_{j i} \times S\left(t_{j}^{B}\right) \times \text { ene }_{j i} \times \text { per }_{j i}\right)
\end{aligned}
$$




\subsection{The Modified Real-Time Fault-Tolerant Task Allocation Schemes}

The mRFTAS employed a modified form of Particle Swarm Optimization (PSO) called discrete Particle Swarm Optimization (dPSO) for the generation of particle position and velocity, this also optimizes the parameter metrics.

PSO is patterned according to the communal behaviour of a flock of birds [10]. It comprises of a swarm of $s$ candidate solutions called particles, which search an $\mathrm{n}$-dimensional hyperspace in the exploration of a global solution ( $n$ represents an optimal number of parameters to be determined). A particle $i$ accepts a position of $X_{i d}$ with a velocity $V_{i d}$ in the $d^{\text {th }}$ which is a hyperspace dimension, $1 \cdot i \cdot s$ and $1 \cdot d \cdot n$. In an objective function every single particle is estimated $f\left(x_{1}\right.$; $x_{2} ; \ldots ; x_{n}$ ), where $f: R^{n} \rightarrow R$. Therefore, its cost (fitness) for every particle near its global solution is lesser (higher) when you consider any particle that is farther away. PSO succeeds to minimize (maximize) (fitness) cost function [10]. Global-best version of PSO considers positions of particle $i$ has its lowest cost which is stored as (pbestid). Also, it's worth knowing that, gbestd, which showcases position particle best used. Each iteration $k$, velocity $V$, and position $X$ are updated applying by the following Equation 16 and 17 [10] [11].

$$
\begin{gathered}
\mathrm{V}_{i d}(k+1)=w \mathrm{~V}_{i d}(k)+\varphi_{1} r_{1}(k)\left(\text { pbest }_{i d}-X_{i d}\right)+\varphi_{2} r_{2}(k)\left(\text { gbest }_{d}-X_{i d}\right) \\
X_{i d}(k+1)=X_{i d}(k)+V_{i d}(k+1)
\end{gathered}
$$

Here, $\varphi_{1}$ and $\varphi_{2}$ are constants, and $r_{1}(k)$ and $r_{2}(k)$ are random numbers uniformly distributed in $[0,1]$. The process which is update iteratively is repeated until acceptable gbest is attained or a grand number of iterations $k_{\max }$ is gotten[10]. The benefits of PSO over many other optimization algorithms are its ease of implementation and its ability to reach a reasonable good solution quickly [10]. PSO is also more efficient in preserving population diversity to avoid premature convergence issue.

In the PSO method is also employed in mRFTAS scheme in a wireless sensor network environment. The flow chart of the scheme as in Figure 2 shows how a sink node collects tasks firstly, and then it generates a position including its velocity in parallel for one generation of dPSO. Next, it begins a series of operations. After that, it looks at the termination condition whether it has been obtained before the next iteration begins. Otherwise, it publishes tasks and waits. Figure 2 on the right part shows the tasks process execution. If the primary version is finished successfully, no matter the mode of corresponding backup version is active or not, it does not have to be executed [2]. If a backup copy mode is passive, it only executed when there is a failure in the primary copy.

\section{Research Results and Discussion}

This chapter will discuss the simulation experiment carried out on the real-time WSNs scenarios. The simulations were carry out using visual studio 2015 professional. The sensor network, simulation area was 100 by 100 . The number of sensor nodes considered are 100-400 nodes. The total number of tasks considered are 400 tasks. Ten (10) Iteration were obtain and the average of the ten results were obtain for all the parameters under consideration. The obtained results are the plots as shown in subsections 3.1, 3.2 and 3.3.

\subsection{Simulation Results of the Task Execution Time of the Sensor Nodes}

The task execution time of a task is the time taken for the sensor nodes to execute their given task to completion. The simulation starts with consideration of 100 nodes and with increments of 25 nodes at interval until the last, which is 250 nodes. The simulations were carry concurrently, for the RFTAS and the mRFTAS scenarios, and the results obtained compared by the plots of Figure 3.

Figure 3 shows the comparative plots of tasks execution time against the number of nodes for both RFTAS and mRFTAS. The ranges of nodes considered for the simulations are; 100, 125, $150,175,200,225$ and 250 nodes. It can be seen from the graphs, the more the number of sensor nodes employed for the execution of 400 tasks, the lesser the time required by the nodes to complete the entire tasks. It is also evident that the task execution time required by the mRFTAS is less than that required by the RFTAS to complete the given task. 


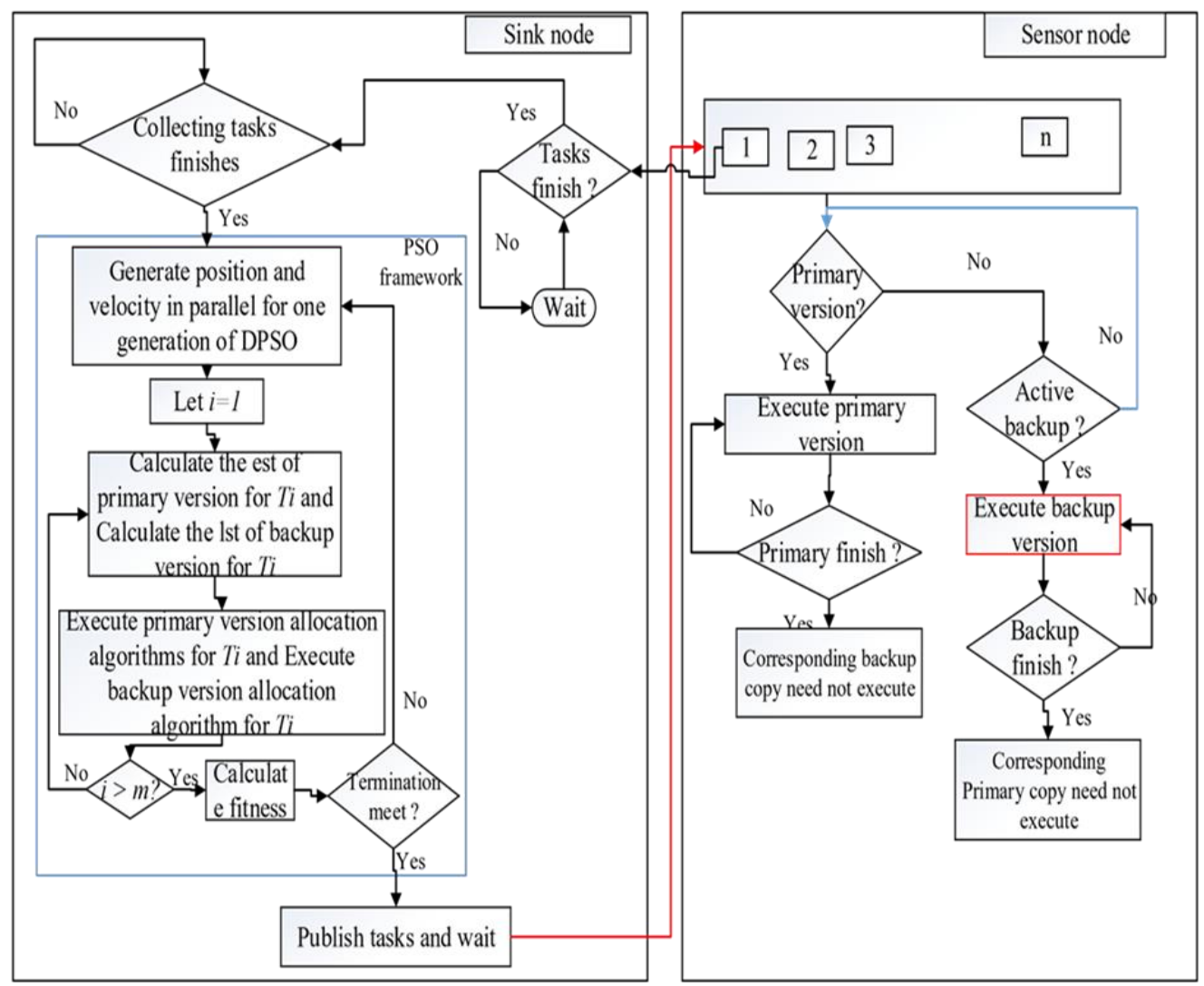

Figure 2. Modified Real-time Fault-tolerant Tasks Allocation Scheme (mRFTAS) Flowchart

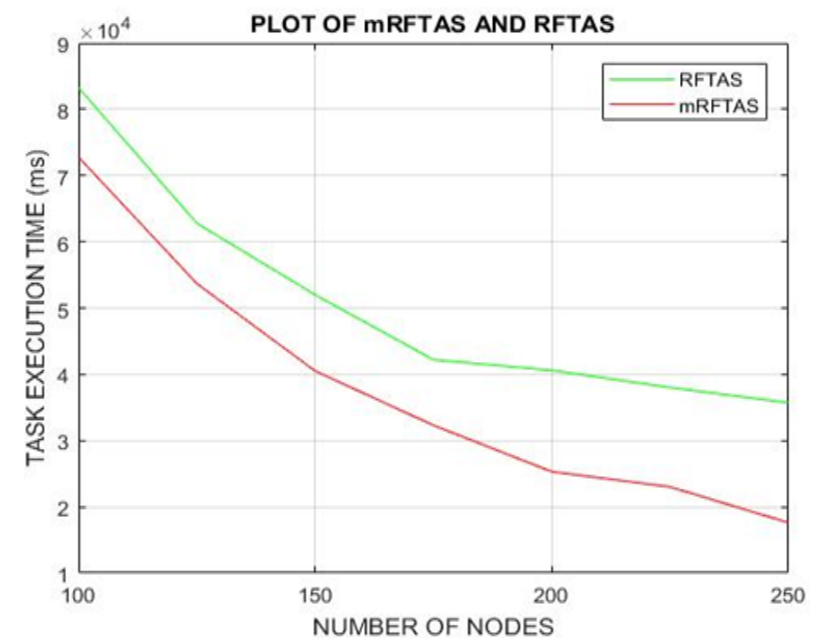

Figure 3. Task Execution Time for mRFTAS and RFTAS

\subsection{Simulation Results of the Reliability Cost of the Sensor Networks}

The simulation starts with consideration of 100 nodes and with increments of 25 nodes at interval until the last, which is 250 nodes. The simulations were carry concurrently, for the RFTAS and the mRFTAS scenarios, and the results obtained compared by the plots of Figure 4 .

Figure 4 is the comparative plot of reliability cost against the number of nodes for the RFTAS and mRFTAS. The ranges of nodes considered for the simulations are; 100, 125, 150, $175,200,225$ and 250 nodes It can be seen that the more the number of sensor nodes employed for the execution of 400 tasks, the lesser the reliability cost associated with the nodes. It is also evident that the reliability cost associated with the mRFTAS is less than that required by the RFTAS. 


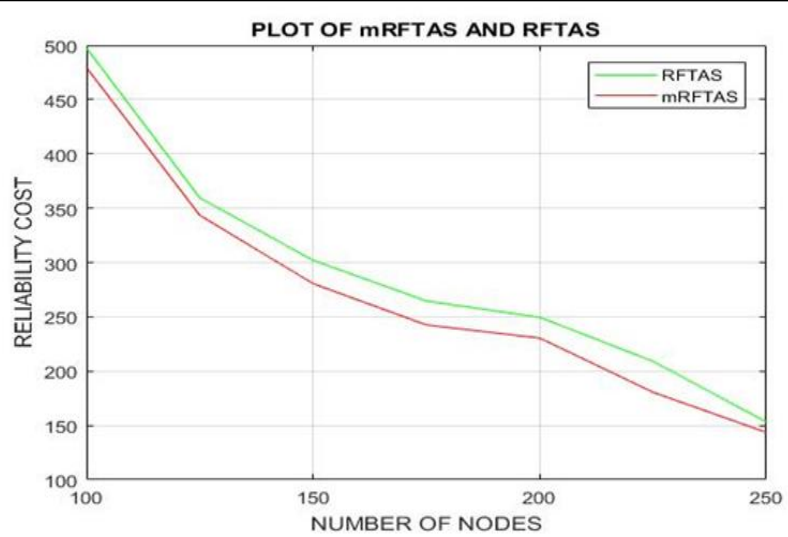

Figure 4. Reliability Cost for mRFTAS and RFTAS

\subsection{Simulation Results of the Network Lifetime of the Sensor Nodes}

The simulations were carry concurrently, for the RFTAS and the mRFTAS scenarios, and the results obtained compared by the plots of Figure 5.

Figure 5 is the comparative plot of a number of failed nodes against the tasks execution time (s) for the RFTAS and mRFTAS. The ranges of time considered for the simulations are; 50, $55,60,65,70,75$ and 80 . It can be seen, the more the time is taken for the execution of the 400 tasks, the more the number of failed nodes. This affects performance and inevitably network lifetime. It is also evident that the mRFTAS had lesser number of failed nodes recorded and invariably a longer network lifetime as compared to the RFTAS.

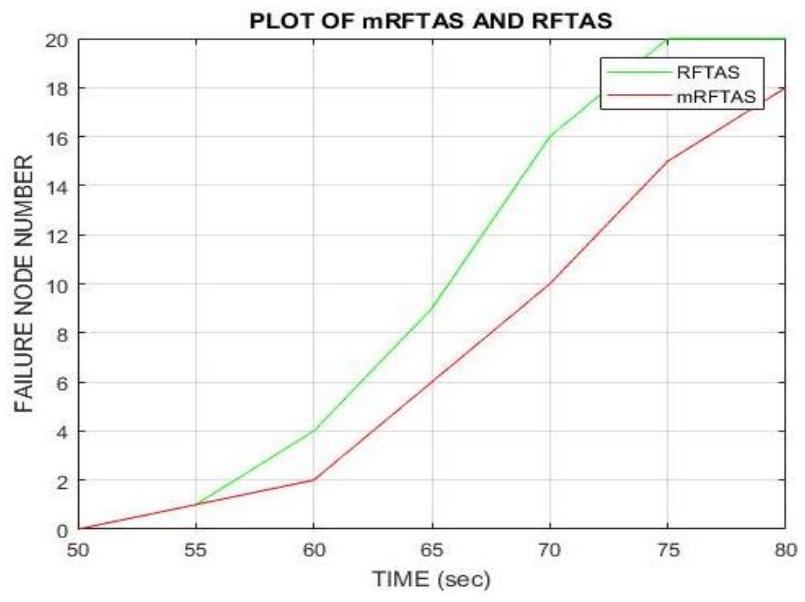

Figure 5. Network Lifetime for mRFTAS and RFTAS

\section{Conclusion}

This paper developed a mRFTAS using active replication backup technique, for addressing the processing time delay and real-time fault tolerance in WSNs by minimizing the time used for task execution and cost in reliability and maximizing network lifetime. The comparisons of performances for both mRFTAS and RFTAS were carried out for time used for task execution, network lifetime and reliability cost. The results obtained from the experimental simulation showed the developed mRFTAS outperformed the RFTAS. From the research carried out, it been realized that in task allocation backup on a system that is real-time and with time constraints, the modified real-time fault-tolerant task allocation scheme is a better technique to be employed. The mRFTAS performance showed an improvement over that of RFTAS when it comes to reducing the time it takes for task execution by $45.56 \%$ and reliability cost of $7.99 \%$ while prolonging the network lifetime by $36.35 \%$.

The following areas of further work are recommended for consideration for future research: The research conducted did not put into consideration malicious nodes presence, thus further work can be carry out by implementing security. The modified Real-time Fault-tolerant task allocation scheme can be modified for minimization of energy consumption in WSNs.

KINETIK Vol. 4, No. 1, February 2019: 45-54 


\begin{tabular}{|c|c|}
\hline Notations & \\
\hline$M$ & $=$ number of tasks \\
\hline Et & $=$ early start time \\
\hline$i \& j$ & = allocated tasks \\
\hline Ist & $=$ latest start time of backup task \\
\hline$t_{i}^{B}$ & = backup task copy \\
\hline$t_{i}^{P}$ & $=$ primary task copy \\
\hline$f_{i}^{P}$ & $=$ finish time of primary copy of task \\
\hline$d_{i}$ & $=$ deadline of $t_{i}$ \\
\hline $\mathrm{P}\left(t_{i}^{B}\right), \mathrm{P}\left(t_{i}^{P}\right)$ & $=$ the nodes $t_{i}^{B}$ and $t_{i}^{P}$ are allocated \\
\hline$S\left(t_{i}^{B}\right)$ & $=$ mode of $t_{i}^{B}$ \\
\hline$l_{i}$ & $=$ laxity of $t_{i}^{P},\left(l_{i}=d_{i}-f_{i}^{P}\right)$. \\
\hline Ene & $=$ energy, $i \& j$ are allocated tasks \\
\hline
\end{tabular}

\section{References}

[1] C. Chen, W. Guo, and G. Chen, "A New Task Allocation Algorithm Based on Dynamic Coalition in WSNs," 2012 IEEE 26th International Parallel Distributed Processing Symposium Workshops \& PhD Forum, Pp. 1243-1248, 2012.

[2] W. Guo, J. Li, G. Chen, Y. Niu, and C. Chen, "A PSO-Optimized Real-Time Fault-Tolerant Task Allocation Algorithm in Wireless Sensor Networks," IEEE Transactions on Parallel Distributed Systems, Vol. 26, No. 12, Pp. 3236-3249, 2015.

[3] W. Guo, Y. Chen, and G. Chen, "Dynamic Task Scheduling Strategy with Game Theory in Wireless Sensor Networks," New Math. Nat. Comput., Vol. 10, No. 03, Pp. 211-224, 2014.

[4] W. Guo, N. Xiong, H.-C. Chao, S. Hussain, and G. Chen, "Design And Analysis Of SelfAdapted Task Scheduling Strategies In Wireless Sensor Networks," Sensors, Vol. 11, No. 12, Pp. 6533-6554, 2011.

[5] P. Suganya and N. Jayanthi, "Sensor Task Reassignment in Wireless Sensor Networks Using Bio-Inspired Algorithm," Int. J. Futur. Innov. Sci. Eng. Res., Vol. 2, No. 1, Pp. 217, 2016.

[6] M. Priyanka, S. Anisha, and S. P. R, "Vlsi Design for a Pso-Optimized Real-Time FaultTolerant," Vol. 11, No. 13, Pp. 8226-8230, 2016.

[7] X. Zhu, X. Qin, and M. Qiu, "QoS-Aware Fault-Tolerant Scheduling for Real-Time Tasks on Heterogeneous Clusters," IEEE Transactions on Computers, Vol. 60, No. 6, Pp. 800-812, 2011.

[8] X. Zhu, J. Zhu, M. Ma, and D. Qiu, "QAFT: A QoS-Aware Fault-Tolerant Scheduling Algorithm for Real-Time Tasks in Heterogeneous Systems," Proc. - 2010 13th IEEE International Conference Computational Science and Engineering (CSE), Pp. 80-87, 2010.

[9] Q. Han and T. Wang, "Enhanced Fault-Tolerant Fixed-Priority Scheduling of Hard Real-Time Tasks on Multi-Core Platforms,"Pp. 21-30, 2015.

[10] S. M. Islam, M. Anisur, R. Reza, and A. Kiber, "Wireless Sensor Network using Particle Swarm Optimization,"Pp. 1-5, 2013.

[11] R. S. Vaishnavi and M. Sukumar, "Survey on Task Allocation using Particle Swarm Optimization in Wireless Sensor Network," International Journal of Innovative Research in Computer and Communication Engineering, Vol. 3297, No. 11, 2015. 
KINETIK Vol. 4, No. 1, February 2019: 45-54 